\title{
Bound state properties from the functional renormalization group
}

\author{
Reinhard Alkofer, ${ }^{1}$ Axel Maas, ${ }^{1}$ Walid Ahmed Mian, ${ }^{1,2}$ Mario Mitter, ${ }^{3}$ Jordi París-López, ${ }^{1,2}$ \\ Jan M. Pawlowski, ${ }^{2,4}$ and Nicolas Wink ${ }^{2}$ \\ ${ }^{1}$ Institute of Physics, NAWI Graz, University of Graz, Universitätsplatz, 5, 8010 Graz, Austria \\ ${ }^{2}$ Institut für Theoretische Physik, Universität Heidelberg, \\ Philosophenweg 16, 69120 Heidelberg, Germany \\ ${ }^{3}$ Physics Department, Brookhaven National Laboratory, Upton, New York 11973, USA \\ ${ }^{4}$ ExtreMe Matter Institute EMMI, GSI, Planckstr. 1, D-64291 Darmstadt, Germany
}

(Received 8 November 2018; published 28 March 2019)

\begin{abstract}
We discuss an approach for accessing bound state properties, like mass and decay width, of a theory within the functional renormalization group approach. An important cornerstone is the dynamical hadronization technique for resonant interaction channels. The general framework is exemplified and put to work within the two-flavor quark-meson model. This model provides a low-energy description of the dynamics of two-flavor QCD with quark and hadronic degrees of freedom. We compare explicitly the respective results for correlation functions and observables with first principle QCD results in a quantitative manner. This allows us to estimate the validity range of low energy effective models. We also present first results for pole masses and decay widths. Next steps involving real-time formulations of the functional renormalization group are discussed.
\end{abstract}

DOI: 10.1103/PhysRevD.99.054029

\section{INTRODUCTION}

The efforts of determining bound state properties in a quantum field theoretical approach date back to the seminal work of Bethe and Salpeter [1,2]. Despite considerable progress in our understanding of bound states and their properties in quantum field theories, the precise computation of their properties and, subsequently also the computation of spectra in general, remains one of the biggest challenges today. Motivated by the immense significance of reliable predictions of these quantities in essentially all areas of physics, there are quite a number of ongoing investigations in this field. This applies in particular to QCD because confinement makes only the composite states of quarks and gluons, the hadrons, experimentally accessible.

The study of highly relativistic bound states has also been hampered by the fact that almost all quantitative nonperturbative methods rely on the Euclidean formulation of quantum field theory. This implies that for determining bound state properties, the results for correlation functions have either to be continued to Minkowski space or have to be extracted from potentially subleading exponential tails of correlation functions. To this end, we note that the existence

Published by the American Physical Society under the terms of the Creative Commons Attribution 4.0 International license. Further distribution of this work must maintain attribution to the author(s) and the published article's title, journal citation, and DOI. Funded by SCOAP ${ }^{3}$. of stable bound states imply poles and of scattering states cuts in the correlation functions for timelike momenta. Unstable particles and virtual states create, in addition, further poles in the complex plane. While the lowest excitations typically can be accessed via reconstruction methods, such techniques fail to provide trustable results for the higher resonances. These higher-lying bound states plainly require an exponentially enhanced precision of the imaginary time data. Moreover, by definition, they lie beyond the radius of convergence for Padé-like analytic continuations of the imaginary time results: Their masses are larger than the ground state mass, and the latter provides the lowest-lying pole in exactly this channel.

In the present work, we suggest a functional continuum approach to bound state computations, which is also put in perspective to other functional bound state approaches; for the impressive progress on bound state and general low energy properties on the lattice, we refer the reader to, e.g., [3]. Due to the bound states poles in the correlation functions, these states, and even higher-lying resonances, can be accessed in continuum approaches via the resonant frequency or momentum structures of higher-order correlation functions of the fundamental degrees of freedom (d.o.f.) as, e.g., quarks and gluons in QCD. In case of gauge theories, physical states will only appear in gauge-invariant channels. However, an understanding of the nonperturbative properties of the elementary correlation functions might necessitate the consideration of bound states in unphysical channels; see, e.g., [4-6]. 
The correlation functions that feature a pole at the bound state masses satisfy Dyson-Schwinger equations as all correlation functions of a theory; for respective reviews, see, e.g., [7-9]. It has been exactly the achievement of Bethe and Salpeter to realize that employing a Laurent expansion of the respective correlation function around the bound state pole, its nonlinear and in general inhomogeneous Dyson-Schwinger equation (DSE) can be reduced to a linear and homogeneous equation, the Bethe-Salpeter equation (BSE), for a simpler quantity, the Bethe-Salpeter amplitude [1,2]. Hereby, the most challenging of the remaining complications is to find an approximation to the kernel of this equation, which is on the one hand, treatable and on the other hand, keeps the most important symmetries of the underlying physics intact. The formulation and discussion of a systematic improvement of employed truncations for the kernel can be found in [9] and references therein. It has to be noted that by far the most used truncation in QCD and hadron physics, a generalized rainbow-ladder truncation, is for quite a number of meson and baryon channels quite successful; see, e.g., the recent Refs. [10-16], but fails nevertheless for a large amount of hadron resonances as it can be inferred, for example, for a combined DSE-BSE approach for light mesons at the three-particle irreducible three-loop level [17] or by recent investigations within the Functional Renormalization Group (FRG) $[18,19]$. It has to be emphasized, however, that keeping the most important symmetries at this level intact requires self-consistency of the treatment of a quite large amount of DSEs for the elementary QCD correlation functions together with the bound state BSEs. This leads to an overwhelming degree of complexity in such calculations. Effectively, it also prevents the use of other available input data for QCD correlation functions, as the most sophisticated calculations imply a high degree of sensitivity of the bound state properties on details of the elementary QCD three-point functions. Note also that an FRG approach very close to the BSE-DSE framework has been put forward in [20,21]; for recent works in this direction, see [22,23].

In the current work, we discuss a unified functional renormalization group approach to bound state properties, based on dynamical hadronization, [24-27]; for more details, see Sec. II. Within dynamical hadronization, correlation functions are not treated as in the DSE-BSE approach by partly including the off shell behavior and partly restricting to on shell properties. Instead, both the quark-gluon correlation functions as well as the Bethe-Salpether wave functions (on and off shell) originate from a common effective action. Naturally, such a unified approach resolves the challenge of self-consistent truncations, which is of crucial relevance in the DSE-BSE framework, by construction. Another important ingredient is the fact, that by now the FRG has matured enough to sustain a systematic access to bound state properties.
Here, we initiate this bound state program with a detailed study of the quark-meson model in the light of the structures explained above. We explain the natural embedding of this low-energy model as an effective field theory (EFT) of QCD as formulated in $[18,19,28]$ within the fQCD collaboration [29]. This includes the determination of the EFT couplings directly in QCD from the QCD flows in $[18,19]$ leading to the QCD-assisted quark-meson model. Then we present results for the (Euclidean) momentum dependence of correlation functions relevant for the access to bound states. As so far only the lightest states will be included, an exploratory investigation into the Minkowski realm can still bee done by means of a reconstruction of the real-time meson propagators. This is notwithstanding the problems listed above for reconstructions and going beyond is our next step.

This paper is organized as follows: In Sec. II dynamical hadronization within the FRG is briefly revisited. The adaption of this approach to QCD as well as the motivation for employing the quark-meson model as a low-energy effective theory are discussed in Sec. III. The truncation of the flow equation for the quark-meson model is detailed in Sec. IV. Our results are then discussed in Sec. V. In Sec. VI the next steps which are required to treat QCD are outlined. Our conclusions are presented in Sec. VII. Technical details as well as a comparison of results in different truncation levels are deferred to three appendixes.

\section{THE FRG AND BOUND STATES}

The FRG approach to bound states as outlined in the present work is based on two key ingredients, the calculation of real-time correlation functions and the concept of dynamical hadronization; both of which are briefly revisited in this section.

\section{A. Real-time FRG}

By now real-time versions of the FRG have been developed that allow us to access real-time correlation functions, and, at the same time, making use of the rather well-developed Euclidean correlation functions of the theories at hand, and in particular, QCD; for recent works, see [30-41].

Here, our task is to extend this to dynamically generated bound state properties. To this end, regulators that preserve $S O(1,3)$ Lorentz symmetry are of paramount importance, and we will focus our discussion of the space-time symmetric regulators suggested in [30-33]. Moreover, it is well-studied by now that the rapid decay of the regularized loops in frequency and momentum space is particularly important in approximations for the full system that do not carry the full frequency and momentum of the theory. Alternatively, one can study some questions about real time observables by applying reconstruction methods. Combined with Euclidean FRG input, this option has been 
used in [42-45]. A detailed analysis of the general complex structure of correlation functions as well as the low and high frequency limits leads to optimized reconstruction schemes; see [46]. An application of this novel reconstruction method to QCD spectral functions can be also found in [46].

\section{B. Dynamical hadronization}

Bound states and resonances in QCD and other theories manifest themselves in resonant momentum channels in scattering amplitudes and correlation functions. Typically, in an effective field theory approach, such a channel can be described as the exchange of an effective field d.o.f. that carries the quantum numbers of the resonant channel. This is the well-known Hubbard-Stratonovitch (HS) transformation, originally introduced as an identity transformation for a local four-point interaction of fermions done on the level of a classical action.

The FRG allows us to perform this identity transformation on the level of the full quantum effective action, which avoids the well-known double-counting problems of the HS transformation, if quantum fluctuations are taken into account. This transformation, called dynamical hadronization or more generally dynamical condensation/bosonization has been introduced in [24] and further developed in [25-27]. While originally introduced for the HS-type transformation for a four-point function, it is by now applicable to general and also nonpolynomial field operators [25]. Its applicability to the full effective action is intertwined with the Wilsonian idea of integrating out fluctuations iteratively momentum shell after momentum shell. As it is done as an exact identity transformation at each RG step, it avoids any double counting issue. This is the property that elevates it to an identity transformation of the full quantum effective action.

One of the prominent advantages within such a formulation is the following. Typically, applications of functional methods to strongly correlated systems such as QCD rely on systematic vertex expansions in the absence of small parameters. The "small" parameter behind this systematics is the phase space suppression of the contributions of diagrams with higher order vertices: These vertices have no classical part and are generated by diagrams in the first place and hence, possess for an asymptotically free theory, a rapid decay behavior in momentum space which leads to, after the angular integration, a very efficient suppression of the respective diagrams. This corresponds to a intuitive picture: these $n$-order vertices describe effectively the local interaction of $n$ particles which is phase space suppressed. However, resonances in interaction channels do not have this suppression if their regularized mass is of the same order as the cutoff or below.

Explicitly, for the case of QCD and hadron physics, this implies that the occurrence of bound states or resonances characterized by two or three valence quarks, i.e., the mesons and baryons, reduces scattering vertices of $(2 n)$ or (3n) quarks and antiquarks to that of $n$ mesons or baryons. This counteracts significantly the phase space suppression of the relevant channels. Accordingly, one either goes to a higher order of the vertex expansion in the fundamental fields in QCD or formulates QCD also in terms of these additional effective d.o.f.

We close this discussion with two remarks on dynamical hadronization: First, we emphasize again that dynamical hadronization does not entail the reduction of QCD to a low energy EFT. It is only a convenient and efficient reparametrization of QCD in the dynamical low energy d.o.f. Second, even though the phase space suppression is partially lifted in the presence of resonant interactions, it is also the mass scale of these channels that decides about their relevance for quantum fluctuations. In QCD, these resonances get strong at low, sub-GeV RG scales $k$. Note that the loop momenta in the FRG framework are restricted by the cutoff scale, $p^{2} \lesssim k^{2}$. Hence, all but the lowest lying resonances are already decoupled when they are generated. Accordingly, it is also quantitatively sufficient to consider the dynamical hadronization of the $\sigma$ mode and the pions $\vec{\pi}$.

Applications to QCD can be found in $[18,19,28,47]$. In particular, Refs. [18,19] contain an application of dynamical hadronization on the quantitative level with full momentum dependencies to QCD with to date by far largest set of coupled set of correlation functions in functional methods.

\section{FUNCTIONAL RENORMALIZATION GROUP APPROACH TO QCD}

Here, we give a brief introduction to the FRG approach to QCD. More details can be found in $[18,19,28,48,49]$; a review on applications can be found in [50]; for QCDrelated FRG reviews, see [25,51-54].

\section{A. Functional renormalization group}

The FRG is based on the Wilsonian idea of successively integrating out quantum fluctuations restricted to a given momentum shell $p^{2} \approx k^{2}$, where $k$ is the running infrared cutoff scale of the theory. The flow of the effective action is then governed by the Wetterich equation $[20,55,56]$,

$$
\partial_{t} \Gamma_{k}[\Phi]=\frac{1}{2} \operatorname{Tr} \frac{1}{\Gamma_{k}^{(2)}[\Phi]+R_{k}} \partial_{t} R_{k},
$$

where $\partial_{t}=k \partial_{k}$, and the superfield $\Phi$ contains all fields in the theory at hand. For example, in $N_{f}=2$ flavor QCD with dynamical hadronization of scalar and pseudoscalar quark-anti-quark channels of the four-quark interaction, we have $\Phi=\left(A_{\mu}, c, \bar{c}, q, \bar{q}, \sigma, \vec{\pi}\right)$. This is the case studied in $[18,19,28]$ relevant for us. $\Gamma_{k}^{(2)}$ is the second field derivative of the effective action, and 


$$
\Gamma_{k}^{(n)}[\Phi]=\frac{\delta^{n} \Gamma[\Phi]}{\delta \Phi^{n}}
$$

The trace in (1) sums over all species of fields, internal indices, and momenta, and $R_{k}\left(p^{2}\right)$ is a regulator function that suppresses the propagation of the infrared modes of all fields.

In practice, the flow equation (1) can only be solved within approximations. As argued above, here we rely on a systematic vertex expansion to QCD; for details, we refer to $[18,19,48,49]$. To that end, we expand the effective action in powers of the fields,

$$
\Gamma_{k}=\sum_{n \geq 1} \frac{1}{n !} \int_{p_{1}, \ldots, p_{n}} \Phi_{1}\left(p_{1}\right) \cdots \Phi_{n}\left(p_{n}\right) \Gamma_{k}^{(n)}\left[p_{1}, \ldots, p_{n}\right],
$$

and solve the flow equations for the $\Gamma_{k}^{(n)}$. This also provides a systematic error estimate of the results with the criterion of apparent convergence: The results apparently converge if, when adding higher orders in the vertex expansion, no substantial changes occur. The system has then passed a nontrivial self-consistency check. Note however, that this is but one of the possible self-consistency checks strongly correlated systems have to pass in the absence of a small expansion parameter.

\section{B. Dynamical hadronization}

As already described in Sec. II B, dynamical hadronization facilitates apparent convergence by means of restoring the canonical phase space suppression ordering of $n$-point functions.

In the present case, we use it for introducing an auxiliary scale dependent mesonic field $\phi_{k}=\left(\sigma_{k}, \vec{\pi}_{k}\right)$ with

$$
\partial_{t} \sigma_{k}=\dot{A}_{k} \bar{q} q, \quad \partial_{t} \vec{\pi}_{k}=\dot{A}_{k} \bar{q} i \gamma_{5} \vec{\tau} q,
$$

where the scale dependence $\dot{A}_{k}$ of this transformation can be chosen arbitrarily. This freedom is used to successively absorb the scalar-pseudo-scalar $u$ channel of the four-fermi scattering vertex; for more details, see Appendix A 4. Then, this channel vanishes identically in the four-fermi vertex. Inserting (4) in (1), we are led to a modified flow equation

$$
\left(\partial_{t}+\partial_{t} \phi_{k}[\Phi] \frac{\delta}{\delta \phi_{k}}\right) \Gamma_{k}[\Phi]=\frac{1}{2} \operatorname{Tr} \frac{1}{\Gamma_{k}^{(2)}[\Phi]+R_{k}} \partial_{t} R_{k} .
$$

The auxiliary field can then be interpreted as the resonance of that channel as it carries the physics and the same quantum numbers. Thereby, dynamical hadronization allows for a convenient access to resonances and all associated bound state properties.

\section{QCD-assisted low energy effective theories}

The flow equation for QCD in the Landau gauge with dynamical hadronization of the $\sigma$ and $\vec{\pi}$ four-quark channels is depicted in Fig. 1. When lowering the cutoff scale $k$ successively, the single loops in Fig. 1 are suppressed if the cutoff scale drops below the mass gap of the respective propagators. This has very important consequences.

First of all, the meson loops do not contribute at large cutoff scales: The effective mesonic mass scale drops rapidly for large cutoffs (and momenta) because the respective momentum channels in the four-quark interaction decay rapidly. This in turn leads to an increased importance of the mesonic loops at low cutoff scales. This is in agreement with the pion mass being the smallest mass scale.

Second, the gluon effectively decouples below cutoff scales of $k \lesssim 1 \mathrm{GeV}$ as the gluon is gapped in the Landau gauge with a mass gap of almost one GeV. Hence, for these scales, the gluon loop can be dropped in Fig. 1 for $k \lesssim 1 \mathrm{GeV}$. Moreover, to leading order, the ghost fields only couple to the matter part of QCD via the gluon. Accordingly, they effectively decouple from the matter sector of QCD as well at the same decoupling scale as the gluon. This leaves us with a quark-meson flow equation at low cutoff scales which is depicted in Fig. 2.

In summary, this leaves us with a QCD-assisted low energy effective theory for QCD. Its natural ultraviolet cutoff $\Lambda$ is at almost $1 \mathrm{GeV}$, and the "classical" action $S_{\mathrm{EFT}, \Lambda}$ of this EFT is the full effective action of QCD at this scale, evaluated on the equations of motion for the gauge field and the ghosts: $A_{\mu}=0, c=0=\bar{c}$, and thus,

$$
S_{\mathrm{EFT}, \Lambda}[q, \bar{q}, \phi]=\Gamma_{\mathrm{QCD}, \Lambda}\left[\Phi_{\mathrm{EFT}}\right],
$$

with the superfield

$$
\Phi_{\mathrm{EFT}}=(0,0,0, q, \bar{q}, \phi), \quad \phi=(\sigma, \vec{\pi}) .
$$

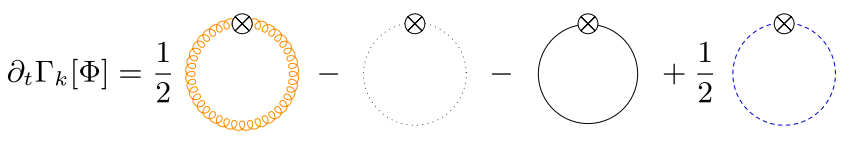

FIG. 1. Functional renormalization group equation for QCD in which the $\sigma-\vec{\pi}$-channel is dynamically hadronized. The lines denote gluons, ghosts, quarks, and mesons, respectively, and represent fully momentum and field dependent propagators. The cross denotes the regulator insertion $\partial_{t} R_{k}(p)$, leading to an effective UV cutoff for modes with $p^{2} \gtrsim k^{2}$.

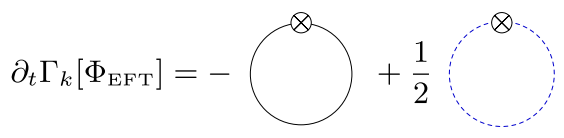

FIG. 2. Functional renormalization group equation for the two-flavor quark-meson model, for the notation $c f$. Fig. 1. 
This concludes the discussion of the QCD embedding of the low energy EFT under investigation here; additional discussions of this issue can be found, e.g., in [28,57-61].

\section{QUARK-MESON MODEL}

Utilizing the previous discussion, we can write down the leading terms of the QCD effective action at a scale where the ghost and gluons are to a large degree already decoupled. Then, the QCD effective action with dynamical hadronization as described in the previous section reduces to a low energy effective theory with quarks and mesons, the quark-meson (QM) model. Bound state considerations in the QM model were amongst the first application of the FRG; see, e.g., [62]; for early reviews, see [51,53,63].

$$
\begin{aligned}
\Gamma_{k}= & \int_{x}\left\{Z_{q, k} \bar{q} \not \partial q+\lambda_{k}\left[\frac{1}{2 N_{f}}(\bar{q} q)^{2}-\left(\bar{q} \gamma_{5} \vec{\tau} q\right)^{2}\right]\right. \\
& +\frac{1}{2} Z_{\sigma, k}\left(\partial_{\mu} \sigma\right)^{2}+\frac{1}{2} Z_{\pi, k}\left(\partial_{\mu} \vec{\pi}\right)^{2}+V_{k}-c \sigma \\
& \left.+h_{k} \bar{q}\left(\mathrm{i} \gamma_{5} \vec{\tau} \cdot \vec{\pi}+\frac{1}{\sqrt{2 N_{f}}} \sigma\right) q\right\} .
\end{aligned}
$$

The effective action (8) consists of three parts: the fermionic and the bosonic ones as well as a Yukawa interaction in between both of them. The latter two, i.e., the second and third line in the effective action (8), result from dynamical hadronization. As for the first line, the quarks' kinetic term appears already in the classical action of QCD. On the other hand, the four-quark interaction is induced by the quark-gluon interaction and is thus created dynamically during the evolution of the scale dependent effective action. Herein, we only consider the scalarpseudo-scalar $u$ channel of the four-fermi vertex, which is by far the most dominant one (see the discussion above). Consequently, the bosonic part contains the propagators for the sigma meson and the pions. These terms as well as the Yukawa interaction are already obtained by a single Hubbard-Stratonovich transformation (see, e.g., Chap. 3 of [64]). Higher-order terms induce self-interactions between the mesons, which are described by the effective potential $V_{k}$. Since isospin symmetry remains unbroken, it is a functional of the $O(4)$-symmetric combination $\rho=\frac{1}{2}\left(\sigma^{2}+\vec{\pi}^{2}\right)$. A nonvanishing expectation value of $\rho$, $\langle\rho\rangle \neq 0$, signals condensation of the sigma meson and thus, spontaneous symmetry breaking. Current quark masses lead via an appropriate shift of the $\sigma$ field to the last term in the second line of the effective action (8). It explicitly breaks $O(4)$ symmetry and effectively alters the expectation value of the sigma meson by tilting the effective potential, which in turn results in a finite mass for the Goldstone modes, i.e., the pions. To summarize, the ansatz (8) for the scale-dependent effective action captures, within a reasonable approximation, the leading behavior of two-flavor QCD at low momenta; a statement tested explicitly in Sec. VA.

In the effective action (8), the wave function renormalization functions, $Z_{\sigma, k}(p), Z_{\vec{\pi}, k}(p)$ and $Z_{q, k}(p)$, the Yukawa coupling $h_{k}\left(p_{1}, p_{2}\right)$, and the four-fermi coupling $\lambda_{k}\left(p_{1}, p_{2}, p_{3}\right)$ are momentum-dependent quantities. Please note that we employ a notation in which momentum conservation is exploited and the three-point vertex $h_{k}$ is a function of two independent momenta, the four-point vertex $\lambda_{k}$ of three momenta. To this end, in the Yukawa coupling, the quark momenta are singled out As already mentioned, the successive Hubbard-Stratonovich transformations in the dynamical hadronization are then used to cancel the flow of the four-fermi coupling; i.e., one implements

$$
\partial_{t} \lambda_{k}(p, p,-p)=0
$$

which yields an additional contribution to the flow of the Yukawa coupling.

In the following, we differentiate between bare quantities and renormalized quantities which are then denoted by a bar. The renormalization conditions are imposed at vanishing momentum (which is the simplest choice) by requiring

$$
\bar{Z}_{\vec{\pi}, k}(p=0)=1 \quad \text { and } \quad \bar{Z}_{q, k}(p=0)=1 .
$$

As a result, all dressed quantities are RG invariant, while the bare ones are not.

Further details about the effective action (8), the solution of the respective flow equation [cf. (1)], and related technical details can be found in Appendix A.

Exploiting the freedom that the four-fermi coupling $\lambda_{k}\left(p_{1}, p_{2}, p_{3}\right)$ can be set to zero at the ultraviolet (UV) cutoff, i.e., for $k=\Lambda_{\mathrm{UV}}$, the four-fermi coupling is absent in all flow equations to be solved in the following. Previous studies in QCD, [19], have shown that the full momentum dependence of the resulting Yukawa vertex can be well approximated by a function of only one momentum upon the substitution rule,

$h_{k}\left(p_{1}, p_{2}\right) \rightarrow h_{k}\left(\sqrt{\frac{1}{4}\left(p_{1}-p_{2}\right)^{2}+\left(p_{1}+p_{2}\right)^{2}}\right)$.

This will be employed in most of the results presented in the next section.

It is also important to note that the quark mass function and the Yukawa coupling are related via the $\sigma$ condensate,

$$
m_{q, k}(p)=\frac{h_{k}(p,-p)}{\sqrt{2 N_{f}}}\langle\sigma\rangle .
$$

The pion decay constant $f_{\pi}$ will in the following be calculated from the $\sigma$ condensate based on an approximation provided by the Gell-Mann-Oakes-Renner relation. 
The accuracy of this strongly simplified way to determine the pion decay constant is sufficient for the purpose of the exploratory investigation presented here.

The initial values for the flow are chosen at the UV cutoff such that physical values for observables are achieved in the infrared $k \rightarrow 0$. This includes setting the pion decay constant to $f_{\pi}=93 \mathrm{MeV}$ and the curvature masses to $\bar{m}_{\pi}=138.7 \mathrm{MeV}, \bar{m}_{\sigma}=500 \mathrm{MeV}$, and $\bar{m}_{q}=297 \mathrm{MeV}$. The specific details on the precise definitions, respectively, the approximations employed for determining the masses are given in the next section. The remaining flow equations can be derived from Fig. 2. This results in the set of equations depicted in Fig. 3. The explicit expressions for the flow equations are deferred to Appendix B.

\section{RESULTS AND DISCUSSION}

We start in Sec. VA with a discussion of the results for Euclidean momenta. The extension of the results to the real time domain for the extraction of bound state properties is discussed in Sec. V B.

\section{A. Solution of the flow equation to Euclidean momenta}

The main results of this section are the momentum dependencies of the propagators as well as the Yukawa coupling. In addition to the physical parameters outlined in Sec. IV, we are considering an additional parameter set where the IR has been fixed to the values of a first principle calculation of QCD correlation functions with the FRG presented in [19]. This comparison has the advantage of testing the validity of low energy effective descriptions of bound states in QCD.

The first quantity of interest from an RG point of view is the flow of the curvature masses evaluated at the flowing minimum of the effective potential, depicted in Fig. 4. The flow of the curvature masses is a key ingredient in all diagrams because it sets the dominant scale in integrands. In the left panel, the result for the quark-meson model with physical IR values is shown. The result is qualitatively similar to previous calculations in the quark-meson model (QM); see, e.g., [65,66].

Since we are mostly interested in the description of bound states, i.e., low-energy effective d.o.f., the comparison to the full QCD calculation is also of particular interest, since the curvature mass determines mainly the scale in the resulting correlation functions. The comparison is depicted in the right panel of Fig. 4 and shows that they agree quite well for small and moderate scales. This demonstrates that the QM model indeed describes the dynamics of low energy QCD correctly. However, in order to achieve the same IR values in the QM setting as in the QCD calculation, the UV cutoff of the theory has to be lowered to $\sim 360 \mathrm{MeV}$; see also [67]. With a higher UV cutoff, it is not possible to decrease the sigma mass further while keeping $f_{\pi}, m_{\pi}$, and $m_{q}$ fixed. This is most likely linked to the triviality of the $\mathrm{O}(\mathrm{N})$ model, which shrinks the values of achievable IR values for a given cutoff when increasing the truncation, which has already been observed in $[33,68]$.

The most significant differences between the QM model and the full QCD calculation is the decoupling of the mesonic masses from the system, which can be seen from comparing both subfigures in Fig. 4: First, the mesonic d.o.f. decouple substantially faster in full QCD. This is also immediately visible when looking at the quark mass in Fig. 5 or at the wave function renormalizations in Figs. 6 and 7. Second, the mass function of the scalar $\sigma$ channel in QCD does not show the dip towards smaller masses in the region where chiral symmetry is restored, which is also related to the steeper rise of the mass function in QCD. This

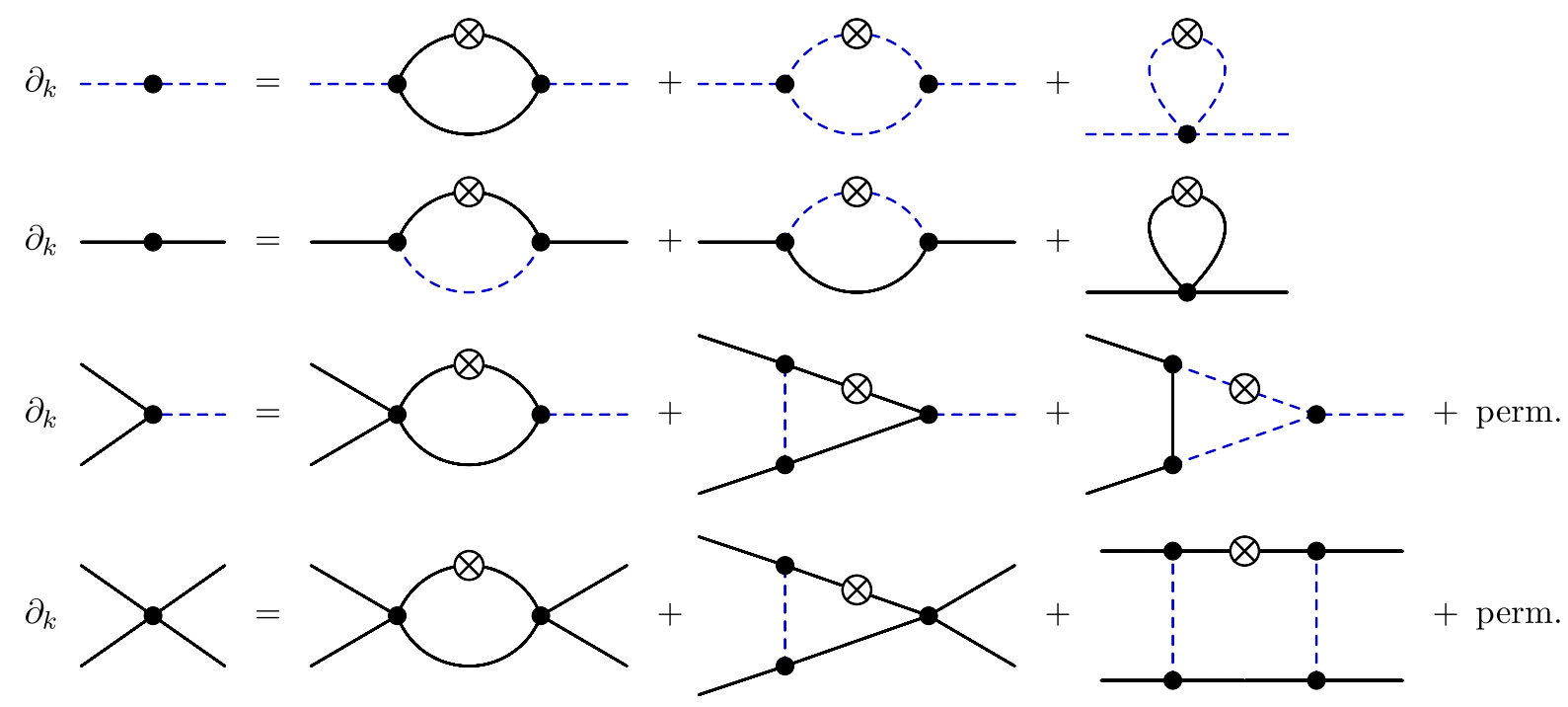

FIG. 3. Flow equations for the propagators, Yukawa coupling, and four-fermi interactions. 

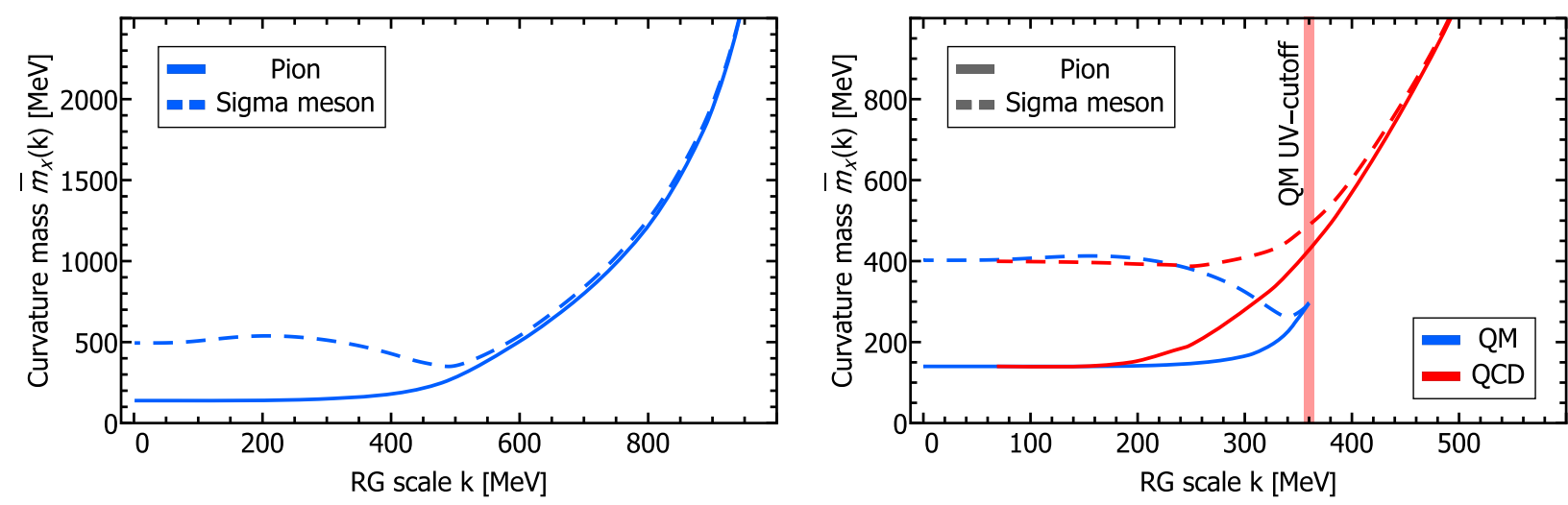

FIG. 4. The flow of the curvature masses for the mesons in the quark-meson model (left panel). The flow is started in the chirally symmetric regime. During the flow, chiral symmetry is broken dynamically, and thus, the masses of pion and sigma run apart in the quark-meson model. In the right panel, the results are compared when the flow of the quark-meson-model is QCD assisted, and compared to those in full QCD from [19].
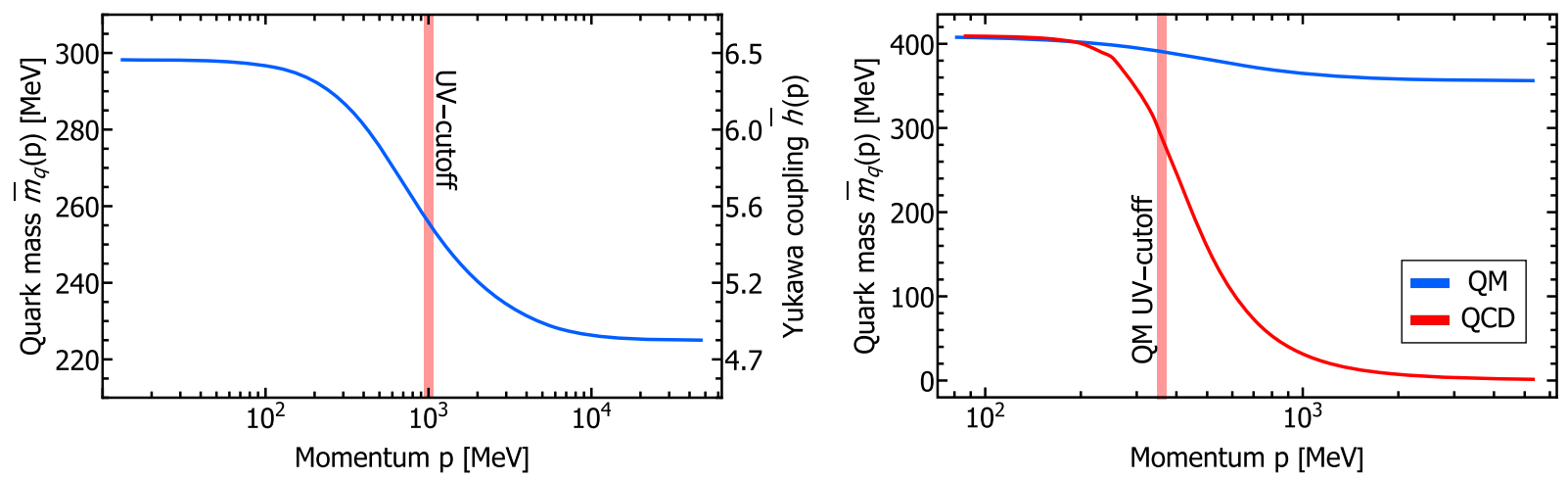

FIG. 5. The momentum-dependent quark mass function in the quark-meson model (left panel). The right panel shows a comparison of the same quantity between the QCD-assisted quark-meson model and QCD.
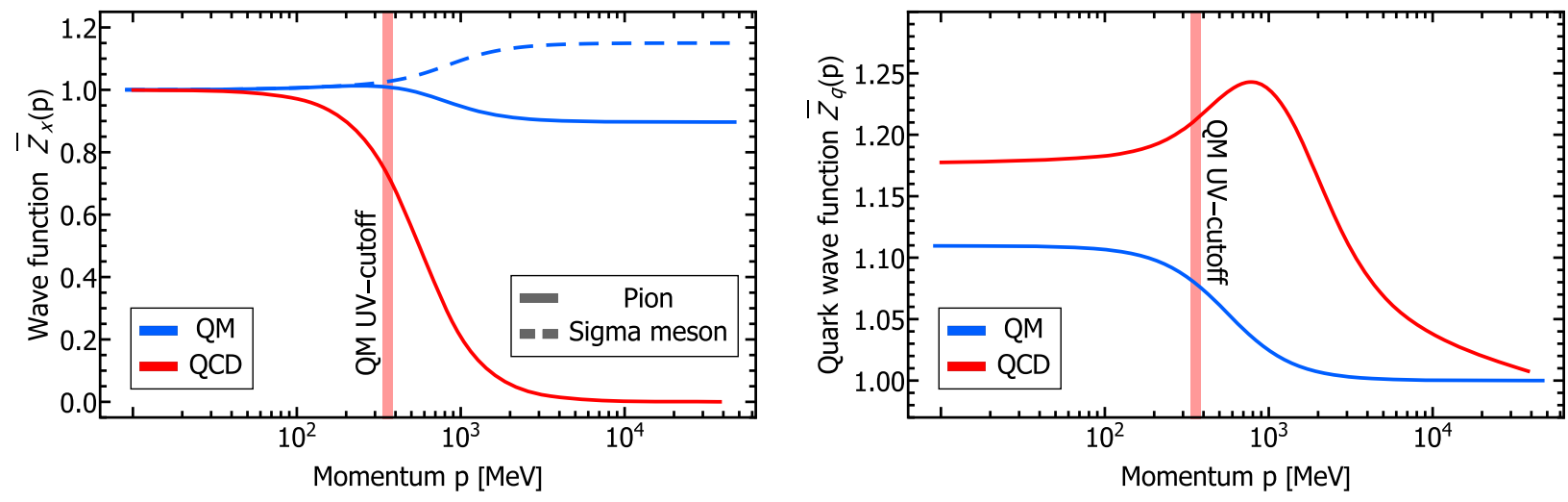

FIG. 6. Comparison between the QCD-assisted QM model and QCD for the momentum-dependent wave function renormalizations for the mesons (left panel) and quarks (right panel).

feature has potentially important consequences for the details of chiral symmetry resaturation at a finite temperature that deserve further investigation. There, the low UV cutoff scale additionally results in a relatively low highest temperature that can be considered due to the thermal range of these models; see [43]. More generally, the necessary modification of the initial effective action for large external scales such as temperature, chemical potential, external 


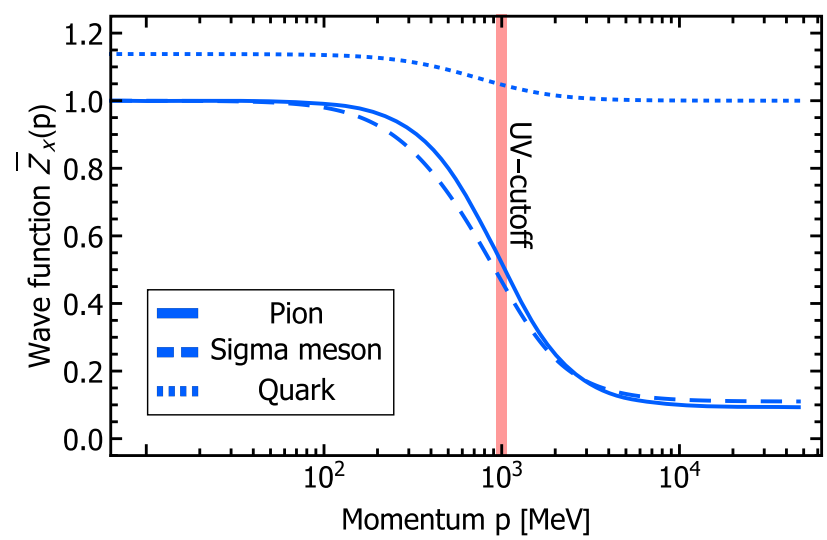

FIG. 7. The momentum-dependent wave function renormalization of the mesons and quarks in the quark-meson model.

background (chromo)magnetic, and electric fields has to be considered; for a detailed discussion, see [69]. Again the systematic inclusion of these modifications is facilitated within a QCD assisted low energy theory.

In all plots, the red vertical line denotes the UV cutoff used in our calculation. The slow decay for large momenta is not very surprising in the quark-meson model as there is no other scale involved which could potentially suppress the mesonic d.o.f. Nevertheless, it is one of the most prominent differences to the full QCD calculation, resulting in a rather small range of momenta, where the quark-meson model properly describes all dynamics of full QCD. However, this does not imply that the description at larger momenta is bad, merely that the question depends strongly on the observable at hand.

\section{B. Continuation to timelike momenta}

The calculation of observables requires in general the knowledge of correlation functions in Minkowski spacetime. In functional methods, this reduces to essentially two distinct options, the direct calculation via analytically continued equations or the numerical analytic continuation. While the former way is preferable, its application to the quark-meson model is postponed to future work. Here, we resort to the latter option, which is for our purposes, i.e., the extraction of the pole masses of the lowest-lying bound states, quite accurate. In addition, numerical analytic continuation is easily possible for this case because the analytic structures of interest are the poles closest to the origin in their respective analytically continued retarded Greens functions; see [46] as well as references therein for a respective detailed discussion. Moreover, it has been already shown in [43] that the momentum dependence of the mesonic propagator is rather mild which also facilitates the reconstruction of the lowest-lying pole. In summary, this allows us to use a Padé based approach, i.e., a rational interpolation. More specifically, we use the Schlessinger's point method [70] to compute the interpolation from a subset of data points.
TABLE I. Curvature mass, pole mass, and decay width, as extracted from the full momentum dependence of the respective propagators. A significant deviation between the pole and curvature mass of the sigma meson is observed. All values are given in $\mathrm{MeV}$.

\begin{tabular}{lccc}
\hline \hline Particle & Curvature mass & Pole mass & Decay width \\
\hline Pion & 138.7 & $137.4 \pm 0.5$ & $0.5 \pm 0.5$ \\
Sigma & 494.5 & $320 \pm 25$ & $36 \pm 5$ \\
\hline \hline
\end{tabular}

The pion is the lightest d.o.f. in the quark-meson model and thus, stable. For the sigma meson, it is found in the present calculation that its pole mass slightly exceeds the two pion decay threshold. Therefore, we expect the mass to be dominated by a pole close to the timelike axis on the second Riemann sheet of the retarded propagator and therefore, accessible within this framework to a good accuracy. The additional numerical error of the reconstruction is checked by computations with different numbers of grid points. The resulting masses and widths are collected in Table I and displayed relative to the curvature masses in Fig. 8. As expected from analytic arguments, the pole mass of the pion agrees well with its renormalized curvature mass $[33,43]$, and its decay width is zero within numerical uncertainties. As outlined above, the sigma meson features in our calculation within the quark-meson model only a small decay width, $\Gamma / M \ll 1$, validating our reconstruction approach. Furthermore, the relative difference between the pole and curvature mass is significant with $\sim 35 \%$. Such an order of magnitude agrees qualitatively with previous studies [33] in which the momentum dependence of the propagators was not fed back into the equations. This has obvious consequences for low energy effective theories of QCD where the mass of the sigma meson is often used to fix the free parameters.

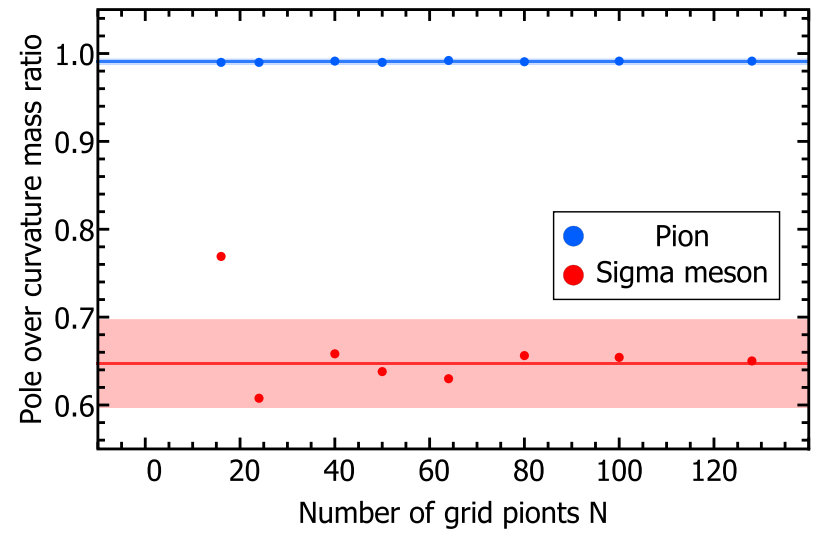

FIG. 8. The ratios of the meson pole masses $m_{\text {pole }}$ and curvature masses $m_{\text {cur }}$ are shown. The ratio is stable for the pion and converges slowly, but eventually, for the sigma as a function of grid points $N$. 


\section{SYSTEMATIC IMPROVEMENTS TOWARDS QCD}

The discussion in Sec. V enables us to systematically improve the current setting towards QCD on a quantitative level. Considering the structure of Fig. 2, or directly in the corresponding equations in Appendix B, it is immediately clear that only three quantities that are not directly derived from the effective potential enter the equations: $\bar{Z}_{\phi}(p)$, $\bar{Z}_{q}(p)$, and $\bar{h}(p, r)$. Please note that we do not need to differentiate between $\bar{Z}_{\pi}(p)$ and $\bar{Z}_{\sigma}(p)$ as their difference is negligible for all points discussed here, $c f$. Fig. 7.

While the difference in the quark wave function $\bar{Z}_{q}(p)$ between the quark-meson model and QCD might appear large in Fig. 6, the values stay practically at unity for all scales, and the bump in QCD at the scale of the gluon mass gap corresponds only to a subleading quantitative correction. Therefore, we can concentrate our discussion on $\bar{Z}_{\phi}(p)$ and $\bar{h}(p, r)$.

Turning to the pion and sigma meson wave functions, significant differences between the quark-meson and the QCD result are observed; see Fig. 7. However, the leading diagrams that generate the momentum dependence are the same in both cases and shown in Fig. 9. As a result, the main difference between the quark-meson and QCD results must be generated by $\bar{h}(p, r)$. In order to confirm this, we can apply the same reasoning to the leading diagrams of the Yukawa coupling in QCD shown in Fig. 10. The first two diagrams, Figs. 10(a) and 10(b), are again self-consistently contained in the quark-meson model. However, the diagram containing a gluon, Fig. 10(c), is not. It is not possible to include this diagram without the knowledge of the gluon

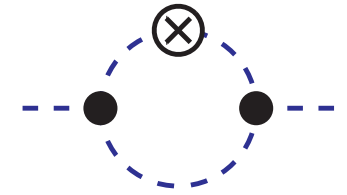

(a) Mesonic contribution

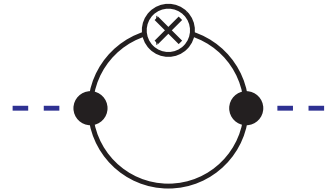

(b) Quark contribution
FIG. 9. The two dominant diagrams contributing to the wave functions of the mesons in QCD (up to permutations of the regulator).

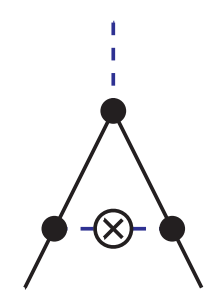

(a) Quark-Meson vertex

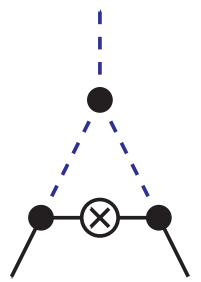

(b) Quark-Meson vertex

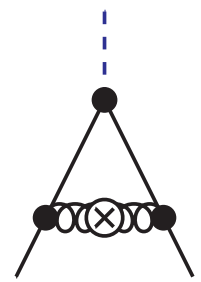

(c) Quark-Gluon vertex
FIG. 10. The three dominant diagrams contributing to the Yukawa coupling in QCD (up to permutations of the regulator). propagator and the quark-gluon vertex building up the one gluon exchange coupling, but QCD-assisted models can be constructed with this input. We conclude from this analysis that the quark-meson model can be systematically improved towards QCD by either including the QCD Yukawa coupling as external input or the gluon exchange coupling in Fig. 10(c). This underlines the strength of the current setting of systematically improvable QCD-assisted low-energy effective theories.

\section{CONCLUSION}

In this work, we have outlined an approach to the calculation of bound states within the Functional Renormalization Group. It is based on the procedure of dynamical hadronization, which comes with the great advantage that the information of bound states can be mapped to lower order $n$-point correlation functions in a systematic manner. Furthermore, this simplifies the procedure of building self-consistent truncations, as all correlation functions are generated from a single master equation, and external input can be incorporated without further problems. These advantages circumvent many of the problems faced when expressing the bound states exclusively in terms of their constituents.

This framework was applied to the pion and sigma mesons within a quark-meson model motivated via dynamical hadronization from QCD and solving the corresponding flow equation for Euclidean momenta. In order to test the validity of our truncation, we have compared the common subset of results with a recent study of the Euclidean system in first principle QCD [19]. While we found at low energies good quantitative agreement, above scales of $\gtrsim 250 \mathrm{MeV}$ qualitative deviations start to appear. These deviations could be traced back to missing contributions in the Yukawa coupling.

The bound state properties of the pion and the sigma meson were accessed from the Euclidean correlators via a suitable Padé approximation extracting hereby the pole masses. While the pole mass of the pion, the lowest lying excitation, agrees very well with the expectation from the Euclidean curvature mass, we found not unexpectedly a significant deviation of $\sim 35 \%$ for the sigma meson.

Most importantly, the here presented approach can and will be systematically extended towards the full bound state spectrum of QCD.

\section{ACKNOWLEDGMENTS}

J. P.-L. and W. A. M. are supported by the FWF Doctoral Program W1203 "Hadrons in vacuum, nuclei and stars" and by the mobility program of the Science Faculty of the University of Graz. M. M. is supported by the FWF Grant No. J3507-N27, the DFG Grant No. MI 2240/1-1, and the U.S. Department of Energy under Contract No. desc0012704. This work is also supported by ExtreMe Matter 
Institute EMMI, the Bundesministerium für Bildung und Forschung Grant No. 05P18VHFCA, and is part of and supported by the DFG Collaborative Research Center SFB 1225 (ISO-QUANT).

\section{APPENDIX A: ADDITIONAL DEFINITIONS AND TECHNICAL DETAILS}

In this section, additional information regarding our ansatz for the effective action (8) and its solution are provided. Furthermore, some additional definitions are introduced, used to keep the flow equations as simple as possible.

\section{Regulator and propagators}

The momentum independent part of the mesonic twopoint function can be obtained from the effective potential,

$$
\begin{aligned}
& \Gamma_{\vec{\pi}}^{(2)}(p=0)=\partial_{\rho} V(\rho), \\
& \Gamma_{\sigma}^{(2)}(p=0)=\partial_{\rho} V(\rho)+2 \rho \partial_{\rho}^{2} V(\rho),
\end{aligned}
$$

which can be obtained from matching the corresponding flow equations. Instead of solving the flow for the full mesonic two-point functions, only the momentum dependent part needs to be considered,

$$
\Delta \Gamma_{i, k}^{(2)}(p)=\Gamma_{i, k}^{(2)}(p)-\Gamma_{i, k}^{(2)}(0), \quad i \in\{\sigma, \vec{\pi}\} .
$$

For the flows of the momentum dependent part, only the polarization diagrams have to be calculated, as the fourpoint functions do not carry any momentum dependence in our truncation, and all tadpoles vanish as a result. The mesonic wave function renormalizations are directly related to the two-point functions by

$$
Z_{i, k}(p)=p^{-2} \Delta \Gamma_{i, k}^{(2)}(p) .
$$

The fermion mass and the Yukawa coupling are related by

$$
m_{q, k}^{2}(p)=\frac{\rho}{N_{f}} h_{k}(p,-p)
$$

As a regulator shape function, we are using a standard exponential regulator,

$$
\begin{aligned}
r_{B}(x) & =\frac{x^{m-1}}{e^{x^{m}}-1}, \\
r_{F}(x)+1 & =\sqrt{r_{B}(x)+1,}
\end{aligned}
$$

resulting in the following full regulators:

$$
\begin{gathered}
R_{i, k}(p)=Z_{i, k}(0) p^{2} r_{B}\left(\frac{p^{2}}{k^{2}}\right), \quad i \in\{\sigma, \vec{\pi}\}, \\
R_{F}(p)=\hat{R}(p) \not p \quad \hat{R}(p)=Z_{q, k}(0) r_{F}\left(\frac{p^{2}}{k^{2}}\right) .
\end{gathered}
$$

We define the following mesonic propagators $(i \in\{\sigma, \vec{\pi}\})$ :

$$
G_{i}(p)=\frac{1}{Z_{i, k}(p) p^{2}+R_{i, k}(p)+\Gamma_{i}^{(2)}(0)} .
$$

In addition, we can define an effective (scalar) quark propagator, entering all loop functions,

$$
\begin{aligned}
G_{q}(p) & =\frac{1}{\left[Z_{r q, k}(p)\right]^{2}+m_{q}^{2}(p)}, \\
Z_{r q, k}(p) & =Z_{q, k}(p)+\hat{R}(p) .
\end{aligned}
$$

\section{Effective potential}

The effective potential $V_{k}(\rho)$ is solved using a Taylor expansion about the IR minimum of the potential, including the explicit symmetry breaking term. The bare expansion point is kept fix, therefore greatly increasing the stability of the equations [65]. It has been verified that the results are independent of the order of the Taylor expansion.

\section{Initial values}

Our initial values are chosen such that the physical values for the quark mass $\bar{m}_{q}=298 \mathrm{MeV}$, the expectation value of the chiral condensate $\sigma_{\min }=93 \mathrm{MeV}$, the pion mass $\bar{m}_{\vec{\pi}}=139 \mathrm{MeV}$, and the curvature mass of the sigma meson $\bar{m}_{\sigma}=495 \mathrm{MeV}$ are obtained. Since the sigma meson is a scattering state in this model, it is not possible to directly assign it a pole mass; therefore, we resorted to the curvature mass, which we fixed slightly higher than the physical mass. More details on the relation between curvature and pole masses can be found in Sec. VB or, e.g., [33]. The flow is initiated at $\Lambda_{\mathrm{UV}}=950 \mathrm{MeV}$, in accordance with our discussion in Sec. III C. The initial potential is assumed to be quadratic

$$
V_{k=\Lambda_{\mathrm{UV}}}=a_{1}\left(\rho-\rho_{0}\right)+\frac{a_{2}}{2}\left(\rho-\rho_{0}\right)^{2},
$$

where $\rho_{0}$ is the expansion point. All other orders of the effective potential are zero. The wave function renormalizations are set to unity at the UV cutoff. For completeness, we also state our initial values for our full truncation considered in this work 


$$
\begin{aligned}
& a_{1}=(2638 \mathrm{MeV})^{2} \quad a_{2}=50 \\
& h_{\Lambda}=18.085 \quad \rho_{0}=28.32 \mathrm{MeV} .
\end{aligned}
$$

The results for the quark and meson masses as well the wave function renormalization functions at $k=0$ do not depend on the UV cutoff if it is changed within reasonable bounds and if the initial values [(A10)] are changed accordingly. This is demonstrated in Figs. 11-13, where these quantities are displayed for two values of the UV cutoff, $\Lambda_{\mathrm{UV}}=950 \mathrm{MeV}$ (black line) and $\Lambda_{\mathrm{UV}}=800 \mathrm{MeV}$.

\section{Dynamical hadronization}

Our dynamical hadronization procedure follows [19]. To this end, we parametrize the scale dependence of the auxiliary field as

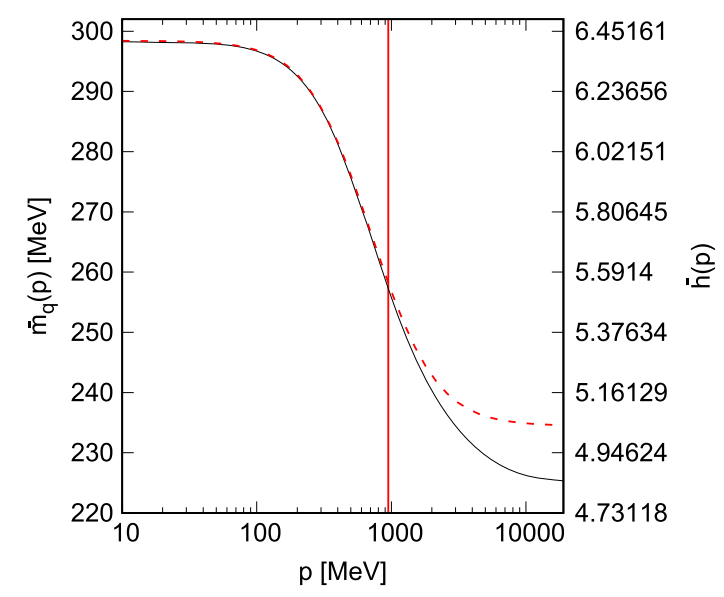

FIG. 11. Momentum dependence of the quark mass, respectively, the Yukawa coupling, at $k=0$ for $\Lambda_{\mathrm{UV}}=950 \mathrm{MeV}$ (black line) and $\Lambda_{\mathrm{UV}}=800 \mathrm{MeV}$ (red dashed line).

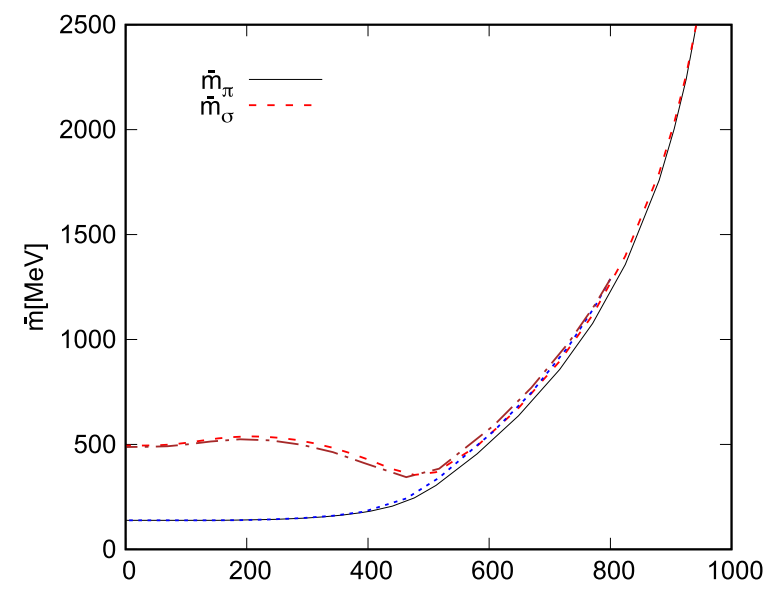

FIG. 12. Scale dependence of the mesonic masses for $\Lambda_{\mathrm{UV}}=$ $950 \mathrm{MeV}$ (black and red full lines) and $\Lambda_{\mathrm{UV}}=800 \mathrm{MeV}$ (blue and brown dashed lines).

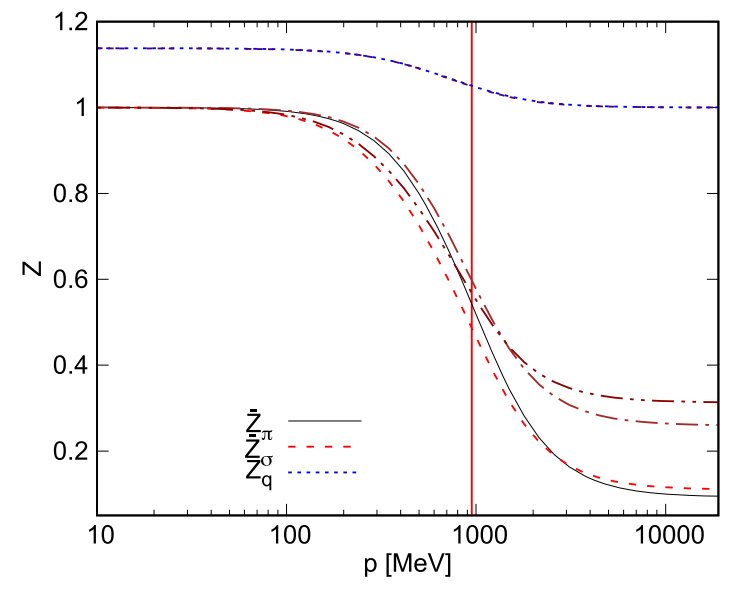

FIG. 13. Wave function renormalization functions at $k=0$ for $\Lambda_{\mathrm{UV}}=950 \mathrm{MeV}$ (pion: black full line, sigma: red dashed line, quark: blue dotted lien) and $\Lambda_{\mathrm{UV}}=800 \mathrm{MeV}$ (pion: brown dotted line, sigma: dark red dotted line, quark: indigo dotted line).

$$
\begin{aligned}
& \dot{\sigma}(p)=\frac{1}{\sqrt{2 N_{f}}} \int_{q} \dot{A}(p-q, q) \bar{q}(p-q) q(q) \\
& \dot{\vec{\pi}}(p)=\int_{q} \dot{A}(p-q, q) \bar{q}(p-q) \mathrm{i} \gamma_{5} \vec{\tau} q(q) .
\end{aligned}
$$

In [19], it was found that a weighted sum between total and relative momenta captures the full momentum dependence quite accurately

$$
\dot{A}\left(p_{1}, p_{2}\right)=\dot{A}\left(\left(p_{1}+p_{2}\right)^{2}+\frac{1}{4}\left(p_{1}-p_{2}\right)^{2}\right) .
$$

As a result, the flow of the corresponding channel in the four-Fermi interaction $\lambda_{k}$ is set to zero,

$$
\begin{aligned}
\partial_{k} \lambda_{k}(p,-p, p) & =\text { Flow } \lambda_{k}(p,-p, p)-\dot{A}\left(p^{2}\right) h_{k}\left(p^{2}\right) \\
\dot{A}\left(p^{2}\right) & =\frac{\text { Flow } \lambda_{k}(p,-p, p)}{h_{k}\left(p^{2}\right)} .
\end{aligned}
$$

\section{APPENDIX B: FLOW EQUATIONS}

For the derivation of the flow equations, DoFun [71] was used. For the subsequent tracing of the equations, we have used FormTracer [72]. We do not state the tadpole (TP) contributions explicitly since we obtain the momentum independent part from (A2). Let us define the relative momenta between the internal and the external momenta $r=q-p$. The contribution to the $\dot{\Gamma}_{\pi, k}^{(2)}(p)$ and $\dot{\Gamma}_{\sigma, k}^{(2)}(p)$ from the quark loop is the same, which is given by 

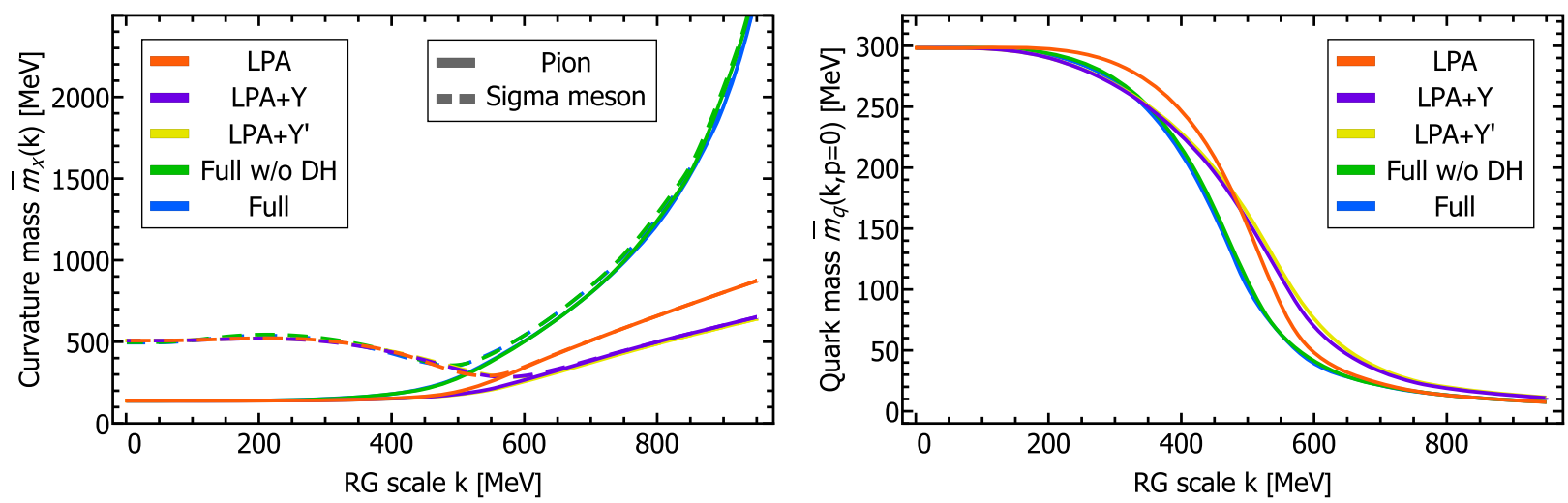

FIG. 14. Flow of the meson masses (left panel) and quark masses at $p=0$ (right panel) shown for different truncations.

$$
\begin{aligned}
\dot{\Gamma}_{\phi, k}^{(2), q}(p)= & \int_{q}\left\{12 \dot{\hat{R}}_{k}^{q}(q) G_{q}^{2}(q) G_{q}(r) h_{k}(r,-q) h_{k}(q,-r)\right. \\
& \times\left[\left(Z_{r q, k}^{2}(q) q^{2}-m_{q, k}^{2}(q)\right) Z_{r q, k}(r)\left(p q-p^{2}\right)\right. \\
& \left.\left.+2 m_{q, k}(q) m_{q, k}(r) Z_{r q, k}(q) q^{2}\right]\right\} .
\end{aligned}
$$

The full flow for the two-point function of the pion is

$$
\begin{aligned}
\dot{\Gamma}_{\pi, k}^{(2)}(p)= & \dot{\Gamma}_{\phi, k}^{(2), q}(p)+\int_{q}\left\{( \Gamma _ { \pi \pi \sigma } ^ { ( 3 ) } ( 0 ) ) ^ { 2 } \left[\dot{R}_{\pi, k}(q) G_{\pi}^{2}(q) G_{\sigma}(r)\right.\right. \\
& \left.\left.+\dot{R}_{\sigma, k}(q) G_{\sigma}^{2}(q) G_{\pi}(r)\right]\right\}+\mathrm{TP},
\end{aligned}
$$

and correspondingly, the full flow of the sigma is

$$
\begin{aligned}
\dot{\Gamma}_{\sigma, k}^{(2)}(p)= & \dot{\Gamma}_{\phi, k}^{(2), q}(p)+\int_{q}\left\{3\left(\Gamma_{\pi \pi \sigma}^{(3)}(0)\right)^{2} \dot{R}_{\pi, k}(q) G_{\pi}^{2}(q) G_{\pi}(r)\right. \\
& \left.+\left(\Gamma_{\sigma \sigma \sigma}^{(3)}(0)\right)^{2} \dot{R}_{\sigma, k}(q) G_{\sigma}^{2}(q) G_{\sigma}(r)\right\}+\mathrm{TP} . \quad(\mathrm{B} 3)
\end{aligned}
$$

The mesonic three point vertices are obtained from the effective potential,

$$
\begin{aligned}
& \Gamma_{\pi \pi \sigma}^{(3)}(0)=\left.\sigma V_{k}^{(2)}[\rho]\right|_{\rho=\rho_{0}}, \\
& \Gamma_{\sigma \sigma \sigma}^{(3)}(0)=\left.\left[3 \sigma V_{k}^{(2)}[\rho]+\sigma^{3} V_{k}^{(3)}[\rho]\right]\right|_{\rho=\rho_{0}} .
\end{aligned}
$$

The flow for the wave function part of the quark propagator reads

$$
\begin{aligned}
\dot{Z}_{q, k}(p)= & -\frac{1}{4 p^{2}} \int_{q}\left\{(p q) \dot{\hat{R}}_{k}^{q}(q) G_{q}^{2}(q) h_{k}(-p, q) h_{k}(-q, p)\right. \\
& \times\left(m_{q, k}^{2}(q)-Z_{r q, k}^{2}(q) q^{2}\right)\left(3 G_{\pi}(r)+G_{\sigma}(r)\right) \\
& +2 G_{q}(r) Z_{r q, k}(r)\left(p q-p^{2}\right) h_{k}(r, p) h_{k}(-p,-r) \\
& \left.\times\left(3 \dot{R}_{\pi, k}(q) G_{\pi}^{2}(q)+\dot{R}_{\sigma, k}(q) G_{\sigma}^{2}(q)\right)\right\}+\mathrm{TP}, \quad
\end{aligned}
$$

and finally, the flow of the Yukawa coupling,

$$
\begin{aligned}
\dot{h}_{k}(p,-p)= & -\frac{1}{4} \int_{q}\left\{2 \dot{\hat{R}}_{k}^{q}(q) G_{q}^{2}(q) h_{k}(-p,-q) h_{k}(q, p)\right. \\
& \times h_{k}(q,-q) Z_{r q, k}(q) q^{2}\left(3 G_{\pi}(r)-G_{\sigma}(r)\right) \\
& +G_{q}(r) h_{k}(-p,-r) h_{k}(r, p) h_{k}(r,-r) \\
& \left.\times\left(3 \dot{R}_{\pi, k}(q) G_{\pi}^{2}(q)-\dot{R}_{\sigma, k}(q) G_{\sigma}^{2}(q)\right)\right\} \\
& -\dot{A}(p,-p) \frac{\Gamma_{\phi}^{(1)}(0)}{\sigma} .
\end{aligned}
$$

The flow of $\lambda_{k}$ has a very lengthy expression and thus is not noted here.

\section{APPENDIX C: TRUNCATIONS}

In this section of the Appendix, we discuss details on the different truncations analyzed within the quark-meson model and compare them to the full case. All truncations are set to get the same IR physics. The results are shown in Figs. 14-16.

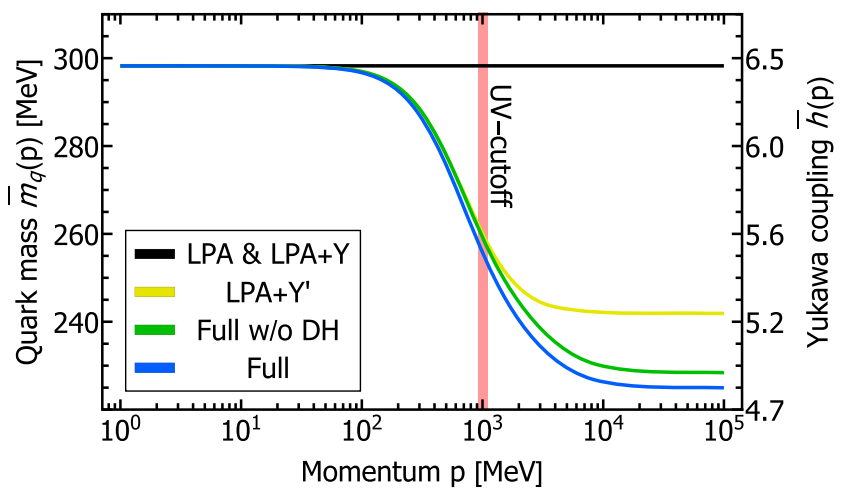

FIG. 15. The momentum dependence of the quark mass (left axes) and Yukawa coupling (right axes) for $k=0$, and different truncations are depicted. The vertical red line shows the value of the UV cutoff. 

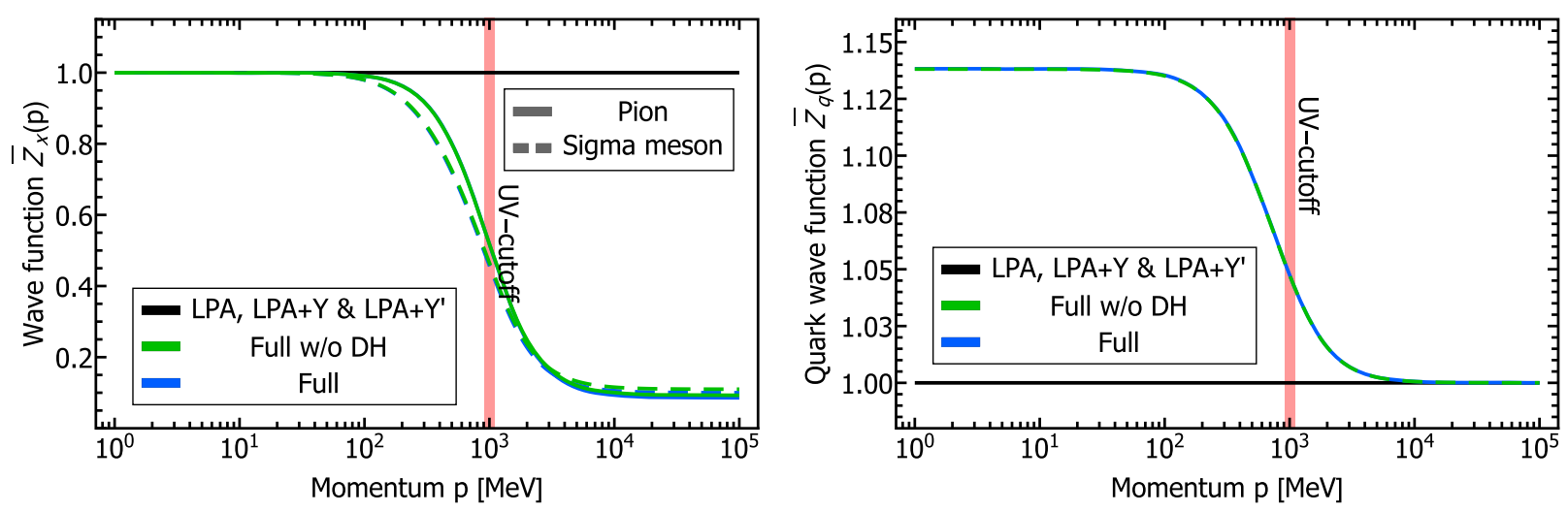

FIG. 16. The momentum-dependent wave function renormalizations for the meson (left panel) and quarks (right panel) is plotted for $k=0$ and different truncations.

In total, we consider four additional truncations

(i) LPA: The wave functions are set to unity, the Yukawa coupling is fixed to a constant and the rebosonization is ignored.

(ii) LPA + Y: The $k$ dependence of the Yukawa coupling is additionally taken into account.

(iii) $\mathrm{LPA}+\mathrm{Y}^{\prime}$ : The momentum dependence of the Yukawa coupling is additionally taken into account.

(iv) Full w/o DH: The full wave functions are taken additionally into account; this corresponds to the full truncation without the rebosonization procedure.
Turning to the running of the curvature masses, shown in Fig. 14, the most notable effect is the faster decoupling of the mesons at larger scales, hence getting closer to the behavior in full QCD, while the running of the quark mass is mostly unaffected. The momentum dependence of the Yukawa coupling, shown in Fig. 15, is below the UV cutoff, essentially independent of the truncation as long as it is calculated. A similar result is found for the wave functions, shown in Fig. 16. These findings fit to the discussion about the different contributions generating momentum dependencies in Sec. VI.
[1] E. E. Salpeter, Phys. Rev. 84, 1226 (1951).

[2] E. Salpeter and H. Bethe, Phys. Rev. 84, 1232 (1951).

[3] S. Aoki et al., Eur. Phys. J. C 77, 112 (2017).

[4] A. C. Aguilar, D. Binosi, and J. Papavassiliou, Phys. Rev. D 95, 034017 (2017).

[5] A. C. Aguilar, D. Binosi, C. T. Figueiredo, and J. Papavassiliou, Eur. Phys. J. C 78, 181 (2018).

[6] N. Alkofer and R. Alkofer, Phys. Lett. B 702, 158 (2011).

[7] R. Alkofer and L. von Smekal, Phys. Rep. 353, 281 (2001).

[8] A. Bashir, L. Chang, I. C. Cloet, B. El-Bennich, Y.-X. Liu, C. D. Roberts, and P. C. Tandy, Commun. Theor. Phys. 58, 79 (2012).

[9] H. Sanchis-Alepuz and R. Williams, J. Phys. Conf. Ser. 631, 012064 (2015).

[10] G. Eichmann, H. Sanchis-Alepuz, R. Williams, R. Alkofer, and C. S. Fischer, Prog. Part. Nucl. Phys. 91, 1 (2016).

[11] T. Hilger, M. Gómez-Rocha, A. Krassnigg, and W. Lucha, Eur. Phys. J. A 53, 213 (2017).

[12] C. Chen, B. El-Bennich, C. D. Roberts, S. M. Schmidt, J. Segovia, and S. Wan, Phys. Rev. D 97, 034016 (2018).

[13] F. E. Serna, B. El-Bennich, and G. Krein, Phys. Rev. D 96, 014013 (2017).

[14] J. Carbonell, T. Frederico, and V. A. Karmanov, Phys. Lett. B 769, 418 (2017).
[15] A. Stadler, S. Leitão, M. T. Peña, and E. P. Biernat, Few Body Syst. 59, 32 (2018).

[16] A. Castro, E. Ydrefors, W. de Paula, T. Frederico, J. H. De Alvarenga Nogueira, and P. Maris, arXiv:1901.04266.

[17] R. Williams, C. S. Fischer, and W. Heupel, Phys. Rev. D 93, 034026 (2016).

[18] M. Mitter, J. M. Pawlowski, and N. Strodthoff, Phys. Rev. D 91, 054035 (2015).

[19] A. K. Cyrol, M. Mitter, J. M. Pawlowski, and N. Strodthoff, Phys. Rev. D 97, 054006 (2018).

[20] U. Ellwanger, Z. Phys. C 62, 503 (1994).

[21] U. Ellwanger and C. Wetterich, Nucl. Phys. B423, 137 (1994).

[22] A. Jakovác and A. Patkós, arXiv:1811.09418.

[23] A. Jakovac and A. Patkos, arXiv:1902.06492.

[24] H. Gies and C. Wetterich, Phys. Rev. D 65, 065001 (2002).

[25] J. M. Pawlowski, Ann. Phys. (Amsterdam) 322, 2831 (2007).

[26] S. Floerchinger and C. Wetterich, Phys. Lett. B 680, 371 (2009).

[27] S. Floerchinger, Eur. Phys. J. C 69, 119 (2010).

[28] J. Braun, L. Fister, J. M. Pawlowski, and F. Rennecke, Phys. Rev. D 94, 034016 (2016). 
[29] J. Braun, L. Corell, W.-J. Fu, C. Huang, M. Leonhardt, M. Mitter, J. M. Pawlowski, M. Pospiech, F. Rennecke, R. Wen, C. Scheider, N. Wink, S. Yin (fQCD Collaboration).

[30] S. Floerchinger, J. High Energy Phys. 05 (2012) 021.

[31] J. M. Pawlowski and N. Strodthoff, Phys. Rev. D 92, 094009 (2015).

[32] N. Strodthoff, Phys. Rev. D 95, 076002 (2017).

[33] J. M. Pawlowski, N. Strodthoff, and N. Wink, Phys. Rev. D 98, 074008 (2018).

[34] K. Kamikado, N. Strodthoff, L. von Smekal, and J. Wambach, Eur. Phys. J. C 74, 2806 (2014).

[35] R.-A. Tripolt, N. Strodthoff, L. von Smekal, and J. Wambach, Phys. Rev. D 89, 034010 (2014).

[36] R.-A. Tripolt, L. von Smekal, and J. Wambach, Phys. Rev. D 90, 074031 (2014).

[37] C. Jung, F. Rennecke, R.-A. Tripolt, L. von Smekal, and J. Wambach, Phys. Rev. D 95, 036020 (2017).

[38] T. Yokota, T. Kunihiro, and K. Morita, Prog. Theor. Exp. Phys. 2016, 073 D01 (2016).

[39] Z. Wang and P. Zhuang, Phys. Rev. D 96, 014006 (2017).

[40] T. Yokota, T. Kunihiro, and K. Morita, Phys. Rev. D 96, 074028 (2017).

[41] M. Bluhm, Y. Jiang, M. Nahrgang, J. M. Pawlowski, F. Rennecke, and N. Wink, Nucl. Phys. A982, 871 (2019).

[42] M. Haas, L. Fister, and J. M. Pawlowski, Phys. Rev. D 90, 091501 (2014).

[43] A. J. Helmboldt, J. M. Pawlowski, and N. Strodthoff, Phys. Rev. D 91, 054010 (2015).

[44] F. Rose, F. Léonard, and N. Dupuis, Phys. Rev. 91B, 224501 (2015).

[45] R. A. Tripolt, I. Haritan, J. Wambach, and N. Moiseyev, Phys. Lett. B 774, 411 (2017).

[46] A. K. Cyrol, J. M. Pawlowski, A. Rothkopf, and N. Wink, SciPost Phys. 5, 065 (2018).

[47] H. Gies and C. Wetterich, Phys. Rev. D 69, 025001 (2004).

[48] A. K. Cyrol, L. Fister, M. Mitter, J. M. Pawlowski, and N. Strodthoff, Phys. Rev. D 94, 054005 (2016).

[49] A. K. Cyrol, M. Mitter, J. M. Pawlowski, and N. Strodthoff, Phys. Rev. D 97, 054015 (2018).

[50] J. M. Pawlowski, Nucl. Phys. A931, 113 (2014).
[51] J. Berges, N. Tetradis, and C. Wetterich, Phys. Rep. 363, 223 (2002).

[52] H. Gies, Lect. Notes Phys. 852, 287 (2012).

[53] B.-J. Schaefer and J. Wambach, Phys. Part. Nucl. 39, 1025 (2008).

[54] J. Braun, J. Phys. G 39, 033001 (2012).

[55] C. Wetterich, Phys. Lett. B 301, 90 (1993).

[56] T. R. Morris, Int. J. Mod. Phys. A 09, 2411 (1994).

[57] G. Papp, B.-J. Schaefer, H. Pirner, and J. Wambach, Phys. Rev. D 61, 096002 (2000).

[58] P. Springer, J. Braun, S. Rechenberger, and F. Rennecke, EPJ Web Conf. 137, 03022 (2017).

[59] J. Braun, M. Leonhardt, and M. Pospiech, Phys. Rev. D 96, 076003 (2017).

[60] J. Eser, F. Divotgey, M. Mitter, and D. H. Rischke, Phys. Rev. D 98, 014024 (2018).

[61] J. Eser, F. Divotgey, and M. Mitter, arXiv:1902.04804.

[62] D. Jungnickel and C. Wetterich, Phys. Rev. D 53, 5142 (1996).

[63] D. U. Jungnickel and C. Wetterich, in The Exact Renormalization Group. Proceedings, Workshop, Faro, Portugal, 1998 (1998), pp. 41-113, https://inspirehep.net/record/ 482851.

[64] R. Alkofer and H. Reinhardt, Lect. Notes Phys., M: Monogr. 33, 1 (1995).

[65] J. M. Pawlowski and F. Rennecke, Phys. Rev. D 90, 076002 (2014).

[66] F. Rennecke and B.-J. Schaefer, Phys. Rev. D 96, 016009 (2017).

[67] F. Rennecke, The Chiral Phase Transition of QCD, Ph. D. thesis, Heidelberg University, 2015.

[68] G. Markó, U. Reinosa, and Z. Szép, Nucl. Phys. B913, 405 (2016).

[69] J. Braun, M. Leonhardt, and J. M. Pawlowski, arXiv: 1806.04432.

[70] L. Schlessinger, Phys. Rev. 167, 1411 (1968).

[71] M. Q. Huber and J. Braun, Comput. Phys. Commun. 183, 1290 (2012).

[72] A. K. Cyrol, M. Mitter, and N. Strodthoff, Comput. Phys. Commun. C 219, 346 (2017). 